\title{
Double Microcatheter-Assisted Coiling of a Basilar Artery Fenestration Aneurysm
}

\author{
Atulabh Vajpeyee, MD, Gourav Goyal, MD, Rama Kant, MD, Narendra Mal, MD
}

Sir,

The incidence of basilar artery fenestration is approximately $0.6 \%$ in angiographic studies and more in autopsy studies [1]. The most common site of basilar artery fenestration is proximal part [2]. Basilar artery fenestration aneurysms are very rare, posing unique challenges. Reported incidence of aneurysm at basilar artery fenestration site is 7\% [3]. Surgical treatment of such aneurysm is usually deferred because of risk of lower cranial nerves injuries, difficulty in exploring surgical field and unpaired arterial supply of brain stem. Endovascular treatment is preferable modality though challenging. We report a case of successful treatment of a wide-necked small $(2.5 \times 3 \mathrm{~mm})$ basilar artery fenestration aneurysm with a novel microcatheter -assisted coiling technique using bifemoral approach. A 60-year-old woman presented with headache and vomiting for 3 days. Evaluation revealed diffuse subarachnoid hemorrhage on plain CT scan. Cerebral DSA revealed a ruptured $(2.5 \times 3 \mathrm{~mm})$ broad-neck proximal basilar artery fenestration aneurysm that incorporated both limbs of the basilar fenestration and both distal vertebral arteries (Fig. 1). An excel 10 microcatheter (Boston Scientific, Natick, MA, USA) was deployed into the right limb of the basilar fenestration from the right distal vertebral artery

All authors: GBH Amaerican Hospital Udaipur, Rajasthan, India Correspondence to: Atulabh Vajpeyee, MD, DM, FINS, Consultant interventional neurologist, Advanced neurocare institute, GBH American hospital, Udaipur-Rajasthan.

Tel. $+91.294 .3056000-228$ Fax. +91.294 .2526982$

E-mail: atulabh@gmail.com, atulabh@gbhamericanhospital.com

This is an Open Access article distributed under the terms of the Creative Commons Attribution Non-Commercial License (http://creativecommons.org/licenses/by-nc/3.0) which permits unrestricted non-commercial use, distribution, and reproduction in any medium, provided the original work is properly cited. to support aneurysm neck. Similarly, another excel 10 microcatheter was placed in proximal basilar fenestration aneurysm through left distal vertebral artery. Following microcatheter support from right side, the aneurysm was treated with coil-embolization through another microcatheter in aneurysm sac from left side (Fig. 2). In basilar artery fenestration aneurysm, either stent assisted coiling or balloon assisted coiling is preferred endovascular technique particularly in wide necked aneurysm to prevent occlusion of fenestrated arm or migration of coiling. Preservation of the fenestrated arm is important as angiographically invisible perforators to brain stem may occlude. Fenestrated basilar artery aneurysm has been classified into type A and type B in the literature [4]. Our case is fitting into type $B$ aneurysm. In this rare case, microcatheter

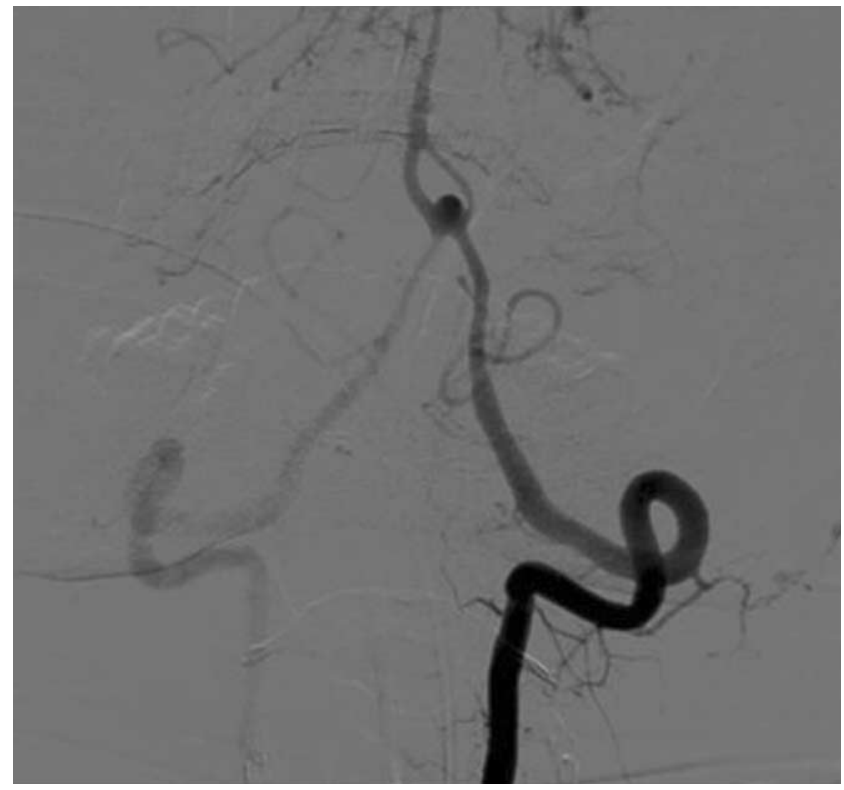

Fig. 1. Pre-treatment, left vertebral artery angiogram. 


\section{Atulabh Vajpeye, et al.}

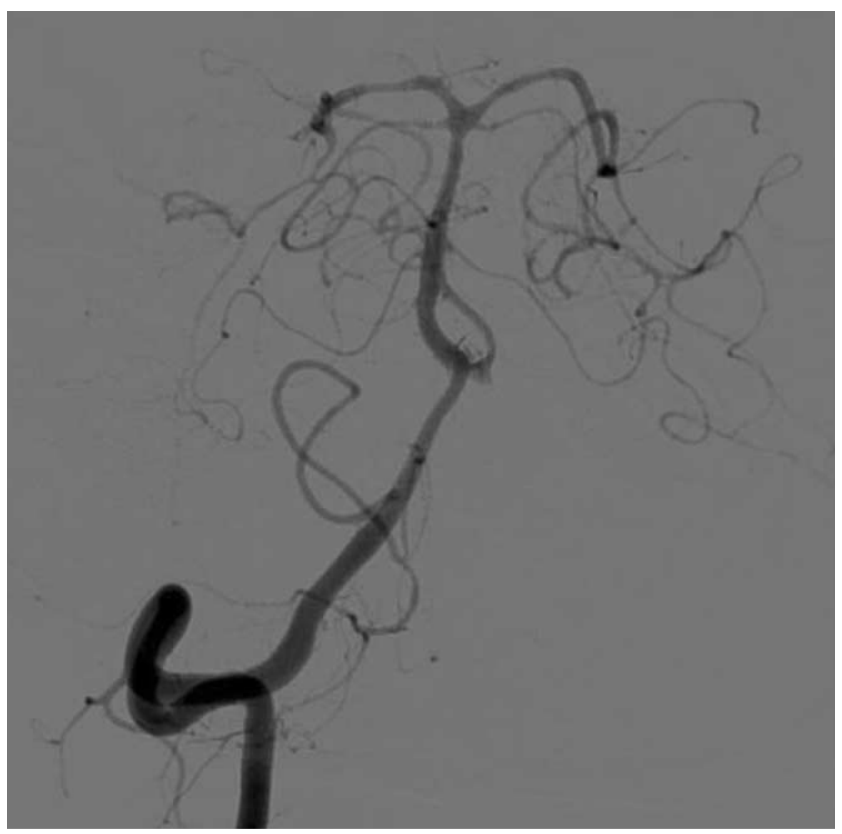

Fig. 2. Post-treatment, right vertebral artery angiogram.

support obviated need of stent which requires antiplatelet use which would have been problematic in view of this being acute subarachnoid hemorrhage. This approach has enabled coil embolization of the aneurysm as well as preservation of both basilar fenestration limbs and both distal vertebral arteries.

Key Words : Basilar artery fenestration aneurysm; Coiling; Aneurysm

Acknowledgement: We acknowledge Staya Narayan for his help.

\section{References}

1. Teal JS, Rumbaugh CL, Bergeron RT, Segall HD. Angiographic demonstration of fenestrations of the intradural intracranial arteries. Radiology 1973;106:123-126

2. Nakasu Y, Nakasu S, Kidooka M, Handa J. Aneurysm at the fenestration of basilar artery: case report. Arch Jpn Chir 1982;51: 344-348

3. Sanders WP, Sorek PA, Mehta BA. Fenestration of intracranial arteris with attention to associated aneurysms and other anomalies. AJNR Am J Neuroradiol 1993;14:675-680

4. Kai Y, Hamada J, Morioka M, Yano S, Fujioka S, Kuratsu J. Endovascular treatment of ruptured aneurysms associated with fenestrated basilar artery. Two case reports. Neurol Med Chir (Tokyo) 2006;46:244-247 